\title{
Baseline Assessment of Primary Healthcare Delivery Through Subcenters of Northern India
}

\author{
Sonu Goel ${ }^{1}$, Madhur Verma ${ }^{2}$, Kamal Kishore $^{3}$, Kritika Upadhyay $^{1}$, Vijai Sharma ${ }^{1}$ \\ 1. Epidemiology and Public Health, Post Graduate Institute of Medical Education and Research, Chandigarh, IND 2. \\ Community and Family Medicine, All India Institute of Medical Sciences, Bathinda, IND 3. Biostatistics, Post Graduate \\ Institute of Medical Education and Research, Chandigarh, IND
}

Corresponding author: Madhur Verma,drmadhurverma@gmail.com

\section{Abstract}

\section{Background}

Our study assessed the status of facilities and services available at the subcenter level and identified the gaps that deter the realization of universal health coverage.

\section{Methods}

A cross-sectional observational study design was used for assessing the subcenters of the Ambala district, Haryana, India with a predesigned, semi-structured tool containing 88 items marked on an ordinal scale. The subcenters were assessed and scored as per their functioning and delivery of various services, their coverage, and quality in terms of various national health programs.

\section{Results}

We found that the essential infrastructure of most of the buildings was average. The types of equipment for antenatal examination, vital medicines, and prominent display boards in the local language were present in all subcenters. The majority of the health workers $(n=27 ; 93 \%)$ successfully demonstrated proper handwashing techniques; $65 \%$ correctly measured blood pressure during an objective structured clinical examination (OSCE), and $48 \%$ could correctly estimate hemoglobin with a hemoglobinometer. Sound knowledge regarding maternal and child healthcare practices and guidelines was noted in $89 \%$ of the health workers.

\section{Conclusions}

The health system of Haryana has scope for improvement in terms of health centers. It is pertinent for the realization of universal health coverage. Therefore, internal audits similar to what we performed must be planned and executed as a regular activity within the framework of existing health systems.

Received 12/09/2019

Review began 12/11/2019 Review ended 12/12/2019 Published 12/16/2019

\section{() Copyright 2019}

Goel et al. This is an open access article distributed under the terms of the Creative Commons Attribution License CC-BY 3.0., which permits unrestricted use, distribution, and reproduction in any medium, provided the original author and source are credited.
Categories: Preventive Medicine, Quality Improvement, Epidemiology/Public Health

Keywords: national health mission, subcenters, supportive supervision, universal health coverage.

\section{Introduction}

India is currently experiencing a rapid transition from communicable to non-communicable diseases, with some evident overlapping [1]. To effectively combat these problems and achieve universal health coverage, service delivery must be improved at the grass-roots level. The Kartar Singh Committee put forward the concept of subcenter (SC) in 1973. The committee proposed the division of primary health centers (PHC) into 16 SCs, to be managed by a team of one male and one female health worker [2]. The Government of India accepted these recommendations and implemented them in the fifth 5-year plan. The National Health Policy, 2017 recommended strengthening the delivery of primary healthcare through the upgrading of the existing SCs and establishment of health and wellness centers as a platform to deliver comprehensive primary healthcare and called for an allocation of two-thirds of the health budget to primary healthcare [3].

The Indian Public Health Standards (IPHS), revised in 2012, lays down the essential and desirable requirements for services, infrastructure, equipment, human resources, and drugs for public health facilities at the SC level [4]. The resources in terms of infrastructure, human resources, and logistics are critical determinants of the service quality delivered by a particular SC. For ethical practice and patient safety, rules, standards, and notifications must be applied to all sectors uniformly. The SCs are constantly criticized for their inability to provide quality health services due to various reasons [5]. In Haryana alone, a shortfall in SCs by approximately $23 \%$ was recently estimated [6].

Supervision was recognized as the most crucial step in improving the SC status and achieving universal health coverage [7]. While supervision can be interactive, traditional supervision focuses on the inspection 
and discovery of faults instead of problem-solving to improve performances [8]. Health workers often receive little advice or guidance on how to improve their results. They are often left unsupervised without a clear definition of objectives. Sometimes the supervisors do not have the technical, managerial, and supervision skills required to assess health facilities adequately. Therefore, they are unable to provide professional advice and appropriate feedback to improve service delivery. Another type of supervision is supportive supervision (SS). It is defined as "a process that promotes quality at all levels of the health system by strengthening the relationships within that system, with an emphasis on identifying and solving problems and contributing to the optimization of the allocation of resources-promotion of high standards, teamwork and better communication in both directions" [9]. The SS creates an enabling environment, facilitates two-way communication, and builds team approaches that improve problem-solving [10]. SS ensures that the tasks are being implemented correctly, focusing on goals and using data optimally for decision-making.

Such supervision leads to an increased sense of ownership, increased work satisfaction, decreased attrition rates, and overall increased work performance among health workers [11]. However, due to the shortage of doctors in our country, SS was neglected until the past few years. Furthermore, it had limited and intermittent activity. SS had been an integral activity in the state of Haryana for some time, but the results were not documented. Moreover, SS was limited to certain domains of the health system, and health facilities were not assessed comprehensively. We have been regularly implementing SS activities in our study area for the past few years. In the present study, we intend to present results from the baseline assessment of the SCs and highlight the problems observed in the infrastructure and service delivery.

\section{Materials And Methods Study settings}

Haryana is one of the northern states of India, with approximately 25 million inhabitants spread across 22 districts [12]. Each district has three to four Community Development Blocks, with each block catering to approximately 80,000-100,000 people. The state health system delivers its services with the help of 2,630 SCs, 486 PHCs, 119 community health centers (CHCs), and 21 district-level hospitals [13]. Antenatal care and immunization services are offered free-of-charge at all levels according to national guidelines. However, Haryana is the only state in India with the dubious distinction to have recorded a rise in maternal deaths in the past two decades [14]. The present assessment was done in Shahzadpur block of Ambala district, which is a field practice area of the Department of Community Medicine and School of Public Health, Post Graduate Institute of Medical Education and Research (PGIMER), Shahzadpur block, Chandigarh. The government health facilities of this area include a CHC, four PHCs, and 29 SCs.

\section{Study design and sampling}

The cross-sectional observational study design was adopted to conduct the study from January to December 2017. Universal sampling was used, wherein all the SCs $(n=29)$ in the study area were included in the study.

\section{Study tool}

A predesigned, semi-structured tool with 88 items and different questions grouped into 11 broad domains was used in this study. The tool collected information on the infrastructure, service delivery, service utilization, quality of services, and operational issues. The tool was adapted from the initial SS tools used by the national health mission department of Haryana. It was further modified by a faculty and two residents of Community Medicine, School of Public Health, PGIMER, Chandigarh. The tool was pretested in two SCs situated in a different area under the department. Minor changes were made, and the tool was finalized; content validation was done by experts from the district administration, such as a Chief Medical Officer of the Ambala district, Block Medical Officer of Shahzadpur, a lady health visitor, and faculty from PGIMER, Chandigarh. The service quality was determined objectively by assessing the clinical competency of the male and female health workers posted at SC with the help of the objective structured clinical examination (OSCE). The OSCE is a versatile multipurpose assessment method for appraising healthcare providers. It evaluates competency based on objective testing through direct observation. Originally, the examination comprises several "stations" in which examinees are expected to perform several clinical tasks within a specified period against specific predetermined criteria essential to demonstrate adequate competence for that particular clinical skill. Moreover, the OSCE evaluates areas that are pertinent to the optimal performance of healthcare providers, such as their ability to record/interpret data, problem-solving, teaching, communication, and handling unpredictable patient behavior, which is otherwise impossible in the traditional clinical examination [15].

\section{Study Protocol}

The final tool was shared with district authorities in advance before undertaking the study. Data were collected as a part of regular SS visits in the health centers of the study area by the residents posted in the Department of Community Medicine. The residents were MD students and had a basic understanding of the hierarchy of public healthcare systems in India and of the IPHS guidelines. The protocol of SS visits to health centers was discussed with them in detail, and they were then asked to gain more information about the 
OSCE. The primary investigator demonstrated the OSCE process during pilot testing so that they felt comfortable with it. After that, a formal schedule for SS visits was communicated with the district health administrators for necessary permissions, and the tour was planned such that it did not disturb the routine work schedule of the centers. Moreover, the schedule was shared with the auxiliary nurse-midwife (ANM) in charge of the SC and the medical officers of the concerned PHC. On the day of the visit, the residents first collected the baseline information about the number of beneficiaries and the logistics situation of the SC. This was followed by a knowledge assessment of the health workers on topics like danger signs of a newborn, high-risk pregnancy, basic treatment guidelines, basket-of-choice approach for family planning, national health programs, and potential beneficiaries. The OSCE assessment was done for different services such as blood pressure (BP) measurement, hemoglobin $(\mathrm{Hb})$ estimation, and protein and albumin content of urine.

\section{Statistical analysis}

The baseline data were entered twice in MS Excel 2013 version (Microsoft, Redmond, WA) and analyzed in the statistical package for social sciences (SPSS) for Windows version 16.0, 2008 version (SPSS Inc., Chicago, IL). All the data collected were validated. It was cross-verified by the principal investigator periodically. Descriptive characteristics were comprehensively assessed and reported in terms of counts, frequencies, and means value as appropriate. Each item was marked on an ordinal scale (good, average, or poor) that measured its effectiveness. Operational definitions were set to label the tool item as good, average, and poor beforehand. If the service or records were well maintained and up-to-date, they were graded as good, if the documents were maintained but not up-to-date, they were marked as average, or otherwise marked poor. The essential skills of health workers were rated based on the scores obtained through OSCE assessment and were labeled as good, average, and poor if the total score obtained was $80 \%-100 \%, 50 \%-80 \%$, and $<50 \%$, respectively. The SCs were evaluated and scored as per the delivery of various national health program services, coverage, and service quality. For this, questions under each domain were assigned one mark. They were then averaged to get a mean score for each domain. This helped us depict the overall performance of the SCs for that domain.

\section{Results \\ Demographic characteristics}

A total of 29 SCs were assessed during the study period. The mean population catered by each SC was 7,112.5 $\pm 1,552$. (range: 5,042-11,820), the mean number of pregnant women enrolled in the SC was $128.07 \pm 39.45$, and the average birth rate in the past financial year was $17 \pm 3.49$. Out of the total two job positions of ANMs at the SC, the first one was hired permanently, while the other one was on a contractual basis. It was observed that contractual job positions were filled in most of the SCs $(n=28)$, while only $50 \%(n=15)$ SCs had their regular positions filled. Only four (13.8\%) regular positions of multi-purpose health worker-male (MPHW-M) were filled against the sanctioned post of 29. All villages had accredited social health activists (ASHAs). The mean distance of SC from its PHC was $8.6 \pm 5.36 \mathrm{~km}$ (range: 0-20 km).

\section{Health facility infrastructure and basic facility assessment}

The building condition was average in 15 (52\%) of 29 SCs (Table 1). In total, 12 (41\%) centers had two rooms, but only one room was being used to provide services. Round-the-clock water supply was present in most of the SCs, but it was not potable, and most of the centers depended on their neighbors. Toilets were nonusable in five $(17 \%)$ centers. Most of the centers $(n=18 ; 62 \%)$ had no provision for uninterrupted electricity supply. Equipment for antenatal examination, essential medicines, and prominent display boards in local language were present in almost all SCs. Record-keeping practices were not uniform, and 14\% of the SCs were not maintaining the record of early registration of antenatal care- and Integrated Management of Neonatal and Childhood Illness (IMNCI)-based assessment of children, whereas 26 SCs (90\%) were not maintaining partograph charts. Health Management Information System (HMIS) data entry was uniformly complete, up-to-date, and accurate. In all the SCs, untied funds were used, while the Annual Maintenance Grant (AMG) utilization rate was less than $50 \%$. 


\section{Cureus}

\begin{tabular}{|c|c|c|c|}
\hline Characteristics & Assessment & & \\
\hline Infrastructure & Good & Average & Poor \\
\hline Condition of the subcenter building & 12 & 15 & 2 \\
\hline Cleanliness and hygiene & 18 & 10 & 1 \\
\hline Rooms used for providing services & 17 & 12 & 0 \\
\hline Toilet availability and condition & 21 & 5 & 3 \\
\hline 24-hour water supply & 20 & 1 & 8 \\
\hline Uninterrupted electricity supply & 9 & 2 & 18 \\
\hline Logistics and supplies & Available and usable & Available but not usable & Not available \\
\hline Equipment for antenatal examination & 29 & 0 & 0 \\
\hline Essential medicines as per the IPHS & 28 & 1 & 0 \\
\hline Tracking bags for routine immunization cards & 15 & 14 & 0 \\
\hline Colored bags for biomedical waste disposal & 29 & 0 & 0 \\
\hline $\begin{array}{l}\text { Display of information, education, and communication } \\
\text { material }\end{array}$ & 24 & 5 & \\
\hline Record-keeping practices & Maintained & Not maintained & Not available \\
\hline Early registration of antenatal care cases & 25 & 4 & \\
\hline Partograph chart & 0 & 3 & 26 \\
\hline Line listing of anemia cases & 23 & 6 & 0 \\
\hline Eligible couple record & 27 & 2 & 0 \\
\hline Routine immunization micro plan & 27 & 2 & 0 \\
\hline IMNCl register & 2 & 27 & 0 \\
\hline Mother and child tracking system work plan & 0 & 0 & 29 \\
\hline HMIS completeness, timeliness, and accuracy & 29 & 0 & 0 \\
\hline Training & 21 & 8 & 0 \\
\hline Financial details & $\begin{array}{l}\text { Available and utilized } \\
>80 \%\end{array}$ & $\begin{array}{l}\text { Available and utilized } \\
80 \% 50 \%\end{array}$ & $\begin{array}{l}\text { Avallable but utilized } \\
<50 \%\end{array}$ \\
\hline Untied funds & 28 & 0 & 1 \\
\hline Annual maintenance grants & 0 & 0 & 29 \\
\hline Monitoring and supervision activities done by & Good & Average & Poor \\
\hline Medical officer & 6 & 0 & 23 \\
\hline Lady health visitor & 5 & 7 & 17 \\
\hline ASHA supervision by ANM & 25 & 2 & 1 \\
\hline
\end{tabular}

\section{TABLE 1: Facility assessment of the subcenters $(n=29)$}

IPHS: Indian Public health standards; IMNCI: Integrated Management of Neonatal and Childhood IIInesses; HMIS: Health Management Information System; ASHA: Accredited Social Health Activist; ANM: auxiliary nurse-midwife

\section{Service delivery}

Services such as antenatal care, home-based postnatal care, childcare during immunization, family planning, and contraception were provided at all the SCs (Table 2). However, merely two-thirds of the SCs provided 


\section{Cureus}

adequate services of essential examination in the first trimester $(n=21 ; 72 \%)$ and almost half in the second and third trimesters $(\mathrm{n}=14 ; 48 \%)$. All births in the past year were through institutional deliveries, and only two (7\%) SCs reported maternal death in the previous financial year. Five (17\%) SCs provided average homebased postnatal care, and only 16 (55\%) SCs provided adequate family planning services after detailed counseling. The vaccination coverage was $>80 \%$ in most of the SCs $(72 \%)$ and was between $50 \%$ and $80 \%$ of their target achievement in the other eight $(27 \%)$ SCs. Only 30\% $(n=10)$ confidently assessed and referred 0 2 month-old infants to a higher level healthcare facility.

Characteristics of the services provided to the

A. Antenatal women

Registration of antenatal care during the first trimester

Essential examination in the first trimester

Essential examination in the second and third trimesters

Institutional delivery

Maternal death in the area

Referral of high-risk pregnancies

Post-natal visits

Family planning services

B. Child health

Vaccination coverage

Sick child (0-2 months) assessed and referred

Sick child ( 2 months -5 years) assessed and referred

\section{Good}

Average

Poor

$\begin{array}{lll}25 & 4 & 0 \\ 21 & 8 & 0 \\ 14 & 15 & 0 \\ 29 & 0 & 0 \\ 27 & \text { NA } & 2 \\ 24 & 2 & 3 \\ 24 & 5 & 0 \\ 16 & 13 & 0\end{array}$

0

$\begin{array}{lll}21 & 8 & 0 \\ 10 & - & 19 \\ 27 & - & 2\end{array}$

TABLE 2: Service delivery characteristics related to maternal and child health observed during supportive supervision of the subcenters $(n=29)$

\section{Assessment of essential skills of the ANMs}

OSCE test was used to assess the knowledge and practices of the ANMs (Table 3). The majority of the health workers ( $n=27 ; 93 \%)$ demonstrated proper handwashing techniques, whereas only two-thirds $(65 \%)$ of the women correctly measured BP as per the OSCE steps, and little less than half (48\%) could correctly demonstrate $\mathrm{Hb}$ estimation with a hemoglobinometer. Most (89\%) of them had sound knowledge regarding maternal and child healthcare practices and guidelines. 


\section{Cureus}

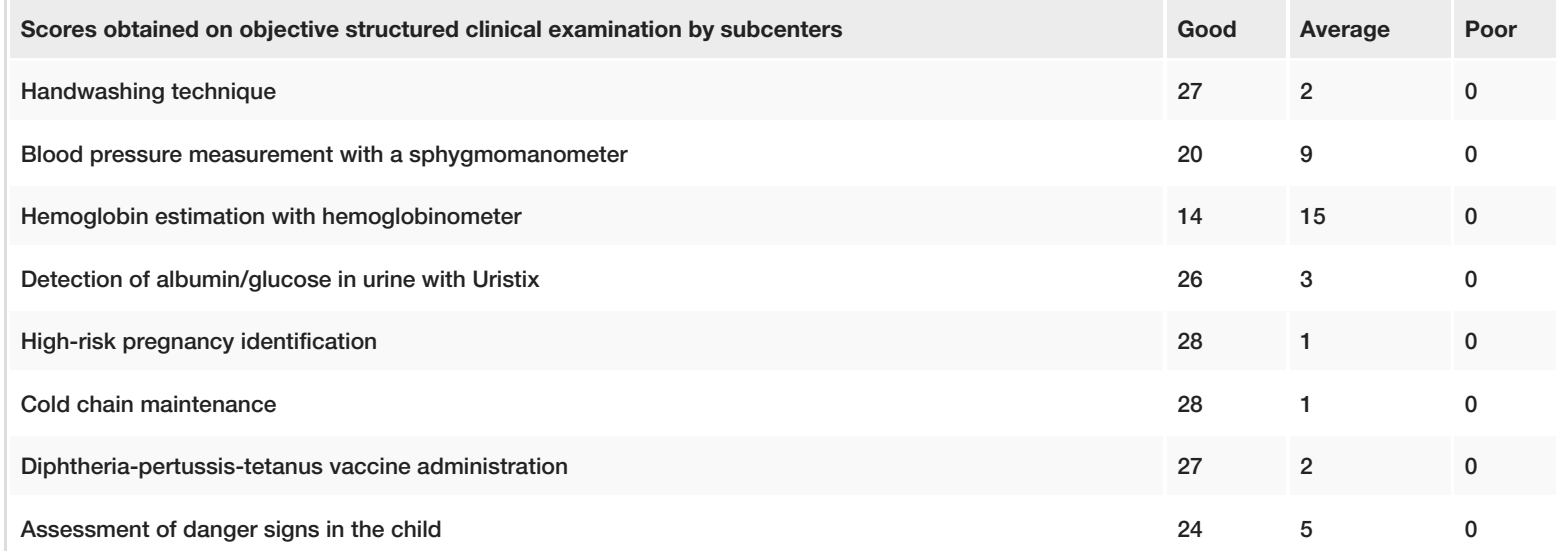

TABLE 3: Assessment of essential skills of health workers posted at the subcenters during baseline assessment visits $(n=29)$

\section{Overall assessment of the SCs}

Table 4 summarizes the overall status of SCs based on the scores given. The SC score was maximum for HMIS, and availability of essential drugs and equipment, whereas the score was low in monitoring and supervision activities and maintenance of financial records. 


\section{Cureus}

\begin{tabular}{|c|c|c|c|c|c|c|}
\hline \multirow{2}{*}{$\begin{array}{l}\text { Parameter/standard (maximum obtainable } \\
\text { score) }\end{array}$} & \multicolumn{3}{|c|}{$\begin{array}{l}\text { Number of SCS under different } \\
\text { categories }\end{array}$} & \multirow{2}{*}{$\begin{array}{l}\text { Mean (SD, } \\
\text { range) }\end{array}$} & \multicolumn{2}{|l|}{ Group score } \\
\hline & Good & Average & Poor & & $(\mathrm{N} \times 29)$ & obtained (\%) \\
\hline Infrastructure and general overview $(n=12)$ & 13 & 14 & 2 & $\begin{array}{l}8.24(2.48,3- \\
12)\end{array}$ & 348 & $239(68.6)$ \\
\hline Essential functioning equipment $(n=6)$ & 29 & 0 & 0 & $\begin{array}{l}5.51(0.50,5- \\
6)\end{array}$ & 174 & 160 (91.9) \\
\hline Essential drugs availability $(\mathrm{n}=2)$ & 28 & 1 & 0 & $\begin{array}{l}1.96(0.18,1- \\
2)\end{array}$ & 58 & $57(98.2)$ \\
\hline Record-keeping practices $(\mathrm{n}=28)$ & 26 & 1 & 0 & $\begin{array}{l}22.03(2.24, \\
17-25)\end{array}$ & 812 & 639 (78.69) \\
\hline Upkeep of financial details $(n=4)$ & 0 & 29 & 0 & $2(0,0)$ & 116 & $58(50)$ \\
\hline IEC activity (boards/posters) at SCs ( $\mathrm{n}=2$ ) & 24 & 5 & 0 & $\begin{array}{l}1.82(0.38,1- \\
\text { 2) }\end{array}$ & 58 & $53(91.3)$ \\
\hline $\begin{array}{l}\text { Provision of reproductive and child health } \\
\text { services }(n=6)\end{array}$ & 16 & 13 & 0 & $\begin{array}{l}4.55(0.82,3- \\
6)\end{array}$ & 174 & $132(75.8)$ \\
\hline HMIS ( $n=6)$ & 29 & 0 & 0 & $6(0,0)$ & 174 & $174(100)$ \\
\hline Training of ANM $(n=2)$ & 21 & 8 & 0 & $\begin{array}{l}1.72(0.45,1- \\
\text { 2) }\end{array}$ & 58 & $50(86.2)$ \\
\hline Knowledge and skills ( $n=16)$ & 28 & 1 & 0 & $\begin{array}{l}14.68(1.36 \\
11-16)\end{array}$ & 464 & $426(91.8)$ \\
\hline Monitoring and supervision $(n=4)$ & 3 & 12 & 14 & $1(1.16,0-4)$ & 116 & $29(25)$ \\
\hline
\end{tabular}

\section{TABLE 4: Categorization of subcenters $(n=29)$ based on supportive supervision parameters/standards}

SC: subcenter; N: number of questions in each domain, also equivalent to maximum obtainable score for that domain; SD: standard deviation; IEC: information, education, and communication; HMIS: Health Management Information System; ANM: auxiliary nurse-midwife

\section{Discussion}

This is among the few studies from India that aimed to assess the adequacy of SCs through SS activities. It mainly focused on assessing the necessary infrastructure and skills that help in effective service delivery by using a comprehensive tool based on IPHS [16]. Moreover, this study documented the knowledge and skill levels of the ANMs recruited under the National Rural Health Mission in 2005 (now renamed as National Health Mission) that prioritized human resources training but produced mixed long-term results [17]. Our study findings reflect the unmet need for supervision and the limited time that supervisors provide in delivering routine immunization services. This initial assessment depicts that most of the SCs still have average infrastructure, and there is room for improvement in record-keeping practices. The quality of service delivery depends mainly on the skills of health workers, which were average in most of the SCs. We stress on different aspects of SS like the regularity of visits by the local officials, mentoring and collaboration, and dialectic communication between supervisors and health workers.

In our study, all the SCs were providing services to more than 5,000 people, which is more than the IPHS norms for SCs, indicating a high burden on the existing SCs for service delivery. The results are similar to the study from Belagavi, Karnataka [18]. All the SCs had their own buildings; but in 2012-2013, only 50\% SCs in Chandigarh and Panchkula were found to be working from their own premises [19]. Although ANMs are supposed to spend most of their time in the community, approximately two-thirds of their total time is spent in center-based activities (conducting an antenatal clinic, well-baby clinic, adolescent clinic, family welfare clinic, and Janani Suraksha Yojana registration). This necessitates the demands of a well-organized SC [20].

Well-organized SCs improve productivity and, hence, job satisfaction [21]. All the SCs had ANMs (either on a regular basis or contractual basis) and ASHAs, which are better than the rural health statistics report 2015, 
which depicted $85 \%$ occupancy of SCs by ANMs. By contrast, only $13.6 \%$ of the SCs had a male health worker (MHW). Moreover, significant gaps were observed in two other districts of Haryana, India, with the MHW post vacant in most of the places $[19,22]$. This perceived low priority given to MHW by the state governments might be due to their ill-defined job responsibilities. MHWs usually spend the majority of their time on the national malaria eradication program and health education activities [23]. In light of the current absence of these workers, further research may evaluate the functioning and effectiveness of the National Vector Borne Disease Control Programme (NVBDCP) activities.

Few SCs were providing adequate services on family planning and maternal and child health. The facilities were similar to those offered in other parts of India [20]. Moreover, the clinical skills exhibited by ANMs were less than satisfactory in most SCs, which is a cause of concern as they are usually the first point of contact at the periphery. Poor clinical skills lead to inappropriate service delivery and, hence, poor program outcomes. Moreover, Singh et al. observed inadequate use of services in the SCs of Madhya Pradesh [24]. Inadequate clinical assessment may lead to a false sense of well-being for most of the people who may need urgent medical care. In this situation, regular supervision using clearly defined and quantifiable indicators can improve service delivery considerably at a modest cost [25]. Another report documented that intense supervision led to a high provider performance, despite initial resistance and extreme variation in individual performance [26]. Financial grants were present only in the form of untied funds in most of the centers that were used entirely. This money is used for repair, payment for tenanted buildings, etc. Nandan et al. (2007-2008) observed that at most SCs, more than 90\% of the untied fund is spent [27]. Round-theclock facilities were not available in any of the SCs as none of the health workers were maintaining headquarters, a typical situation, as observed in another study as well [24]. However, recently, the Government of India has initiated the transformation of SCs into health and wellness centers that can provide essential medical services round the clock. These health facilities will act as a provider of holistic healthcare services package with zero cost to the patient [28].

The results of the skill assessment of ANMs were motivating as they knew the need and purpose of services delivered at the primary healthcare level. For example, most of the health workers knew about hypertension and diabetes mellitus, their implications, and how to measure them. The mean score was comparable with another study, although the skills were average when they demonstrated the process of BP measurement with a sphygmomanometer, $\mathrm{Hb}$ estimation with a hemoglobinometer, and detection of albumin/glucose in urine with Uristix [29]. The health workers were simultaneously taught regarding the processes that were observed to be wrong or missing during the OSCE examination, and they wholeheartedly welcomed it. We need to address the gaps in skills by providing on-job training and demonstrations to health workers at regular intervals.

The study shows that SS emergence paved the way not only for assessing performance but also for monitoring services, evaluating management, and ensuring proper working of health facility supply chains [30]. Moreover, the study has highlighted that budget constraint should not be blamed for the low performance of the SCs. Most of the parameters that can be improved (skill-building, training, recordkeeping practices, and monitoring) are within the scope of the existing means and resources. Moreover, the success of the study can be assessed through the determination of its adherence to the SS principles described in the background section of this paper, such as handholding for quality improvements, strict use of validated tools, and skill assessment against set standards. However, this study had certain limitations as well. In addition to the small sample size, a mixed-method approach could have been used in the study to reveal different aspects regarding the deficiencies from various stakeholders and probable scope for the improvement of service delivery. We could not assess the long-term effect of SS, as it was a cross-sectional study. Future studies should aim to lay down the dashboard indicators that can objectively evaluate the healthcare delivery through SCs and revisit them after a while to look for sustainable changes.

\section{Conclusions}

The present study has highlighted the role of SS in improving the delivery of services in healthcare. Implementation of SS is recommended within the framework of national health systems with similar institutional arrangements for health services organizations. We recommend putting in place supervision services that can provide complementary support mechanisms to health workers and supervisors alike for the overall achievement of universal health coverage. Supervision activities can be strengthened through streamlining supervision protocols to focus less on report checking and more on problem-solving and skill development.

\section{Additional Information \\ Disclosures}

Human subjects: Consent was obtained by all participants in this study. Institutional Ethics Committee, Post Graduate Institute of Medical Education and Research, Chandigarh. issued approval IEC-11/2017-757. The Institutional Ethics Committee approved the study protocol of PGIMER, Chandigarh, India (approval no: IEC-11/2017-757, letter number: PGI/IEC/2017/632). Consent was obtained from all participants before the interview. Animal subjects: All authors have confirmed that this study did not involve animal subjects or tissue. Conflicts of interest: In compliance with the ICMJE uniform disclosure form, all authors declare the 
following: Payment/services info: All authors have declared that no financial support was received from any organization for the submitted work. Financial relationships: All authors have declared that they have no financial relationships at present or within the previous three years with any organizations that might have an interest in the submitted work. Other relationships: All authors have declared that there are no other relationships or activities that could appear to have influenced the submitted work.

\section{Acknowledgements}

We are thankful to the Department of Health, Government of Haryana, India for providing us a conducive environment to conduct this research. We are also thankful to Wallace Academic Editing for editing this manuscript

\section{References}

1. Situation analyses, backdrop to the national health policy . (2017). Accessed: December 3, 2019: https://mohfw.gov.in/sites/default/files/71275472221489753307.pdf.

2. Kartar Singh Committee, 1973. (2015). Accessed: December 3, 2019: https://www.nhp.gov.in/kartar-singhcommittee-1973_pg.

3. Ayushman Bharat - health and wellness center . (2019). Accessed: December 3rd, 2019: https://abhwc.nhp.gov.in/.

4. Indian public health standards. (2012). Accessed: December 3, 2019: https://nhm.gov.in/index1.php? lang=1 \&level=2 \&sublinkid=971 \&lid=154.

5. Reddy NB, Prabhu GR, Sai TS: Study on the availability of physical infrastructure and manpower facilities in sub-centers of Chittoor district of Andhra Pradesh. Indian J Public Health. 2012, 56:290-292. 10.4103/0019557X.106417

6. Report of the Task Force on Comprehensive Primary Health Care Rollout . (2014). Accessed: December 3, 2019: http://nhsrcindia.org/sites/default/files/Report of Task Force on Comprehensive PHC Rollout.pdf .

7. Vasan A, Mabey DC, Chaudhri S, Brown Epstein HA, Lawn SD: Support and performance improvement for primary health care workers in low- and middleincome countries: a scoping review of intervention design and methods. Health Policy Plan. 2017, 32:437-452. https://doi.org/10.1093/heapol/czw144

8. Developing and strengthening community health worker programs at scale . (2013). Accessed: December 3, 2019: http://chwcentral.org/sites/default/files/MCHIP_CHW\%20Ref\%20Guide.pdf.

9. Making supervision supportive and sustainable: new approaches to old problems . (2002). Accessed: December 3, 2019: https://www.usaidassist.org/sites/assist/files/maqno4final.pdf.

10. Improving health worker performance: in search of promising practices . (2006). Accessed: December 3, 2019: https://www.kit.nl/wp-content/uploads/2018/08/1174_Improving-health-workerperformance_Dieleman_Harnmeijer.pdf.

11. Jaskiewicz W, Tulenko K: Increasing community health worker productivity and effectiveness: a review of the influence of the work environment. Hum Resour Health. 2012, 10:38. Accessed: December 3, 2019: 10.1186/1478-4491-10-38

12. Haryana population census data. (2011). Accessed: December 3, 2019: https://www.census2011.co.in/census/state/haryana.html.

13. District-wise availability of health centres in India as on 31st march 2017 . (2017). Accessed: December 10, 2019: https://data.gov.in/resources/district-wise-availability-health-centres-india-31st-march-2017.

14. Reddy H, Pradhan MR, Ghosh R, Khan AG: India's progress towards the Millennium Development Goals 4 and 5 on infant and maternal mortality. WHO South-East Asia J Public Health. 2012, 1:279-289. 10.4103/2224-3151.207024

15. Avortri GS, Nabukalu JB, Nabyonga-Orem J: Supportive supervision to improve service delivery in lowincome countries: is there a conceptual problem or a strategy problem?. BMJ Glob Health. 2019, 11:e001151. Accessed: December 3, 2019: 10.1136/bmigh-2018-001151

16. Indian Public Health Standards (IPHS) guidelines for primary health centres revised . (2012). Accessed: December 3, 2019: https://nhm.gov.in/images/pdf/guidelines/iphs/iphs-revised-guidlines-2012/primayhealth-centres.pdf.

17. Gopalakrishnan s, Immanuel A: Progress of health care in rural India: a critical review of National Rural Health Mission. Int J Community Med Public Health. 2018, 5:4-11. 10.18203/2394-6040.ijcmph20175758

18. Patil SK, Shivaswamy MS: Assessment of sub-centres of Belagavi district according to Indian Public Health Standards 2012 guidelines: a cross sectional study. Natl J Community Med. 2016, 7:749-753. 10.18203/23946040.ijcmph20172153

19. Dhiman A, Goel NK, Walia DK, Galhotra A, Singh N: Assessment of health centers as per Indian Public Health Standards in Chandigarh Tricity, India. Indian J Appl Res. 2014, 3:420-421. 10.15373/2249555X/July2014/133

20. Narayanasamy NS, Lakshminarayanan S, Kumar SG, Kar SS, Selvaraj K: How multipurpose health workers spend time during work? results from a time-and-motion study from Puducherry. Indian J Community Med. 2018, 43:5-9. 10.4103/ijcm.IJCM_276_16

21. Aziz I, Kumar R, Rathore A, Lal M: Working environment and job satisfaction among health professional working at a tertiary care hospital of Pakistan. J Ayub Med Coll Abbottabad. 2015, 27:201-204.

22. Kumar A, Jain RB, Khanna P: Pattern of nutrition and health guidance to adolescents by their teachers and multipurpose health workers in a rural block of district: Jhajjar (Haryana). J Med Nutrition \& Nutraceuticals. 2014, 3:85-88. 10.4103/2278-019X.131959

23. Nair VM, Thankappan KR, Sarma PS, Vasan RS: Changing roles of grass-root level health workers in Kerala, India. Health Policy Plan. 2001, 16:171-179. 10.1093/heapol/16.2.171

24. Singh G, Mathur BP, Chaturvedi S, Rai P, Agarwal D: Poor quality control of the first contact point of community: findings of cross-sectional study on subcentres in district Jhansi. Indian journal of forensic and 


\section{Cureus}

community medicine. 2015, 2:1-6.

25. $\mathrm{COPE}{ }^{\circledR}$ for maternal health services: a process and tools for improving the quality of maternal health services. (2001). Accessed: December 3, 2019: http://bit.ly/34TukCR.

26. WHO recommendations for clinical mentoring to support scale-up of HIV care, antiretroviral therapy and prevention in resource-constrained settings. (2005). Accessed: December 10, 2019: http://www.who.int/hiv/pub/guidelines/clinicalmentoring.pdf.

27. An assessment of utilization of untied fund provided under National Rural Health Mission in Uttar Pradesh . (2008). Accessed: December 3, 2019: http://bit.ly/2rxXubY.

28. Comprehensive primary health care. (2013). Accessed: December 3, 2019: http://nhsrcindia.org/categorydetail/comprehensive-primary-health-care/MjE=.

29. Jelly P, Sharma R. : OSCE vs TEM: different approaches to assess clinical skills of nursing students . Iran J Nurs Midwifery Res. 2017, 22:78-80. 10.4103/ijnmr.IJNMR 10716

30. Kroeger A, Hernandez JM: Health services analysis as a tool for evidence-based policy decisions: the case of the Ministry of Health and Social Security in Mexico. Trop Med Int Health. 2003, 8:1157-1164.

10.1046/j.1360-2276.2003.01143.x 